\title{
Treatment of singularly perturbed differential equations with delay and shift using Haar wavelet collocation method
}

\author{
Arshad Khan and Akmal Raza
}

\begin{abstract}
An efficient Haar wavelet collocation method is proposed for the numerical solution of singularly perturbed differential equations with delay and shift. Taylor series (upto the first order) is used to convert the problem with delay and shift into a new problem without the delay and shift and then solved by Haar wavelet collocation method, which reduces the time and complexity of the system. Further, we apply the Haar wavelet collocation method directly to solve the problems. Also, we demonstrated several test examples to show the accuracy and efficiency of the Haar wavelet collocation method and compared our results with the finite difference and fitted operator finite difference method [11, 29].
\end{abstract}

Keywords. Haar wavelet, collocation grid, singular perturbation, delay, shift

\section{Introduction}

Singularly perturbed differential equations with delay and shift are also known as the singularly perturbed differential-difference equations (SPDDEs) or delay differential equations with small shift, which arises frequently in the modelling of neuronal variability, determination of expected time for generation of action potentials in nerve cell by random synaptic, study of human pupil light reflex, mathematical biology, various model of physiological process and disease, controllability, study of bistable devices and inputs in dendrites. Stein have given delay differential equations model incorporating stochastic effects due to neutral variability and approximate the solution using Monte-Carlo techniques. The standard finite difference method is not sufficient to solve SPDDEs. Many numerical methods like fitted operator finite difference method, finite element subspace method, standard finite difference on a fitted piecewise uniform mesh, exponentially fitted mesh finite difference method based on Il'in-Allen Southwell fitting and non standard finite difference method have been developed for the solution of SPDDEs, refer [10]-[12], [15]-[20], [29], [31].

In this paper, we consider the following SPDDEs

$$
\epsilon^{2} y^{\prime \prime}(t)+a(t) y(t-\delta)+b(t) y(t)+c(t) y(t+\eta)=f(t), \quad t \in(0,1)
$$

Received date: May 6, 2019; Published online: February 21, 2021.

2010 Mathematics Subject Classification. 65M99; 65N35; 65N55; 65L10; 65L12; 34K26; 34K28. Corresponding author: Akmal Raza. 
with the interval conditions

$$
y(t)=\phi(t), \quad \text { on } \quad-\delta \leq t \leq 0,
$$

and

$$
y(t)=\psi(t), \quad \text { on } \quad 1 \leq t \leq 1+\eta,
$$

where $a(t), b(t), c(t), f(t), \phi(t)$ and $\psi(t)$ are sufficiently smooth functions, $\epsilon$ is a perturbation parameter, $0<\varepsilon \ll 1 ; \delta, \eta$ are delay and shifting parameters which depends on $\epsilon$ [20]. If the delay and shift terms are zero, then the SPDDE becomes singularly perturbed differential equations which can be seen in $[2,13,14,19,25,27,32,33]$. Using Haar wavelet we transforms the SPDDEs into a system of algebraic equations.

Due to the piecewise constant nature, compact support, symmetric and orthogonality of the Haar wavelet, it makes the nonsingular sparse matrix with respect to the collocation points while solving the differential equations. Since the Haar wavelet is not differentiable, the solution of second order differential equation is approximated by piecewise quadratic polynomials (second order integration of Haar wavelet) on applying integration approach. Thats why Haar wavelet has less calculation and take less computational time to solve the problem. The theoretical stability of the Haar wavelet can be seen in Debnath and Shah [5]. In case, if we use any other wavelet present in literature such as Legendre wavelet, Hermite wavelet, Laguerre wavelet, Bernoulli wavelet, Chebyshev wavelet and Gegenbauer wavelets, it takes more computational time due to their higher degree polynomial nature and non-symmetricity. These wavelets yield full matrices while solving differential equations on collocation points in both differentiation and integration approaches.

The organization of the paper is as follows. We describe the basic Haar wavelet and integration of Haar wavelet in Section 2. Method to solve SPDDEs is described in Section 3. Further, the convergence analysis of the proposed method and error calculation formula are also described. Several test problems of SPDDEs are solved in Section 4. Maximum absolute errors of each problem are reported and the results are presented in tables.

\section{Haar Wavelet}

The characteristics like symmetry, compact support, flexibility and orthogonality of Haar wavelet allows to approximate any square integrable function at different resolution level. Haar wavelet is the simplest orthonormal wavelet with compact support. This wavelet is the member of the family of similar shaped wavelet of different scales located at different position of time domain [1],[4]-[8, 24, 28]. The Haar wavelet family for $t \in[0,1]$ is defined as follows.

$$
\mathcal{H}_{i}(t)= \begin{cases}1, & \xi_{1}(i) \leq t<\xi_{2}(i), \\ -1, & \xi_{2}(i) \leq t<\xi_{3}(i), \\ 0, & \text { otherwise }\end{cases}
$$

where $i$ indicates the wavelet number and

$\xi_{1}(i)=\frac{k}{m}, \quad \xi_{2}(i)=\frac{k+0.5}{m}, \quad \xi_{3}(i)=\frac{k+1}{m}, \quad m=2^{j}, j=0,1,2, \ldots, J, \quad k=0,1, \ldots, m-1$.

Here $J$ indicates the level of resolution and $k$ represents the translations parameter. Index $i$ is calculated as $i=m+k+1$ which is true for $i \geq 2$. For $i=1$, the Haar wavelet is given by

$$
\mathcal{H}_{1}(t)= \begin{cases}1, & 0 \leq t<1, \\ 0, & \text { otherwise }\end{cases}
$$


Due to lack of differentiability of Haar wavelets, authors move towards integration approach instead of differentiation $[3,9,21,23,26,28,30]$.

The integration $\mathcal{P}_{i}(t)$ of Haar wavelet can be obtained as follows:

$$
\mathcal{P}_{i}(t)=\left\{\begin{array}{lc}
t-\xi_{1}(i), & \xi_{1}(i) \leq t<\xi_{2}(i), \\
\xi_{3}(i)-t, & \xi_{2}(i) \leq t<\xi_{3}(i), \\
0, & \text { otherwise }
\end{array}\right.
$$

Furthermore, the double integration $\mathcal{Q}_{i}(t)$ of Haar wavelet can be obtained as follows:

$$
\mathcal{Q}_{i}(t)= \begin{cases}\frac{1}{2}\left(t-\xi_{1}(i)\right)^{2}, & \xi_{1}(i) \leq t<\xi_{2}(i) \\ \frac{1}{4 m^{2}}-\frac{1}{2}\left(\xi_{3}(i)-t\right)^{2}, & \xi_{2}(i) \leq t<\xi_{3}(i) \\ \frac{1}{4 m^{2}}, & \xi_{3}(i) \leq t<1 \\ 0, & \text { otherwise }\end{cases}
$$

Proceeding in similar manner the $n$th integration of Haar wavelet can be obtained as in [21]-[23], [26] as follows:

$$
I_{n} \mathcal{H}_{i}(t)= \begin{cases}0, & t<\xi_{1}(i), \\ \frac{1}{n !}\left[t-\xi_{1}(i)\right]^{n}, & \xi_{1}(i) \leq t<\xi_{2}(i), \\ \frac{1}{n !}\left[\left(t-\xi_{1}(i)\right)^{n}-2\left(t-\xi_{2}(i)\right)^{n}\right], & \xi_{2}(i) \leq t<\xi_{3}(i), \\ \frac{1}{n !}\left[\left(t-\xi_{1}(i)\right)^{n}-2\left(t-\xi_{2}(i)\right)^{n}+\left(t-\xi_{3}(i)\right)^{n}\right], & \xi_{3}(i) \leq t\end{cases}
$$

Now consider, any square integrable function $f(t) \in L^{2}[0,1]$, which can be approximated by the dilation and translation of Haar wavelet [3, 4] as

$$
f(t)=\sum_{i=1}^{N} a_{i} \mathcal{H}_{i}(t), \quad N=2^{J+1} .
$$

The Haar wavelet coefficients $a_{i}$ are computed by following formula,

$$
a_{i}=\left\langle y(t), \mathcal{H}_{i}(t)\right\rangle=\int_{0}^{1} y(t) \cdot \overline{\mathcal{H}_{i}(t)} d t .
$$

The collocation grid is given as

$$
X(u)=\frac{2 u-1}{m}, \quad u=1,2, \ldots, m .
$$

The matrix of Haar wavelet with respect to the collocation grid is given as

$$
\mathcal{H}=\left(\begin{array}{cccccccc}
1 & 1 & 1 & 1 & 1 & 1 & 1 & 1 \\
1 & 1 & 1 & 1 & -1 & -1 & -1 & -1 \\
1 & 1 & -1 & -1 & 0 & 0 & 0 & 0 \\
0 & 0 & 0 & 0 & 1 & 1 & -1 & -1 \\
1 & -1 & 0 & 0 & 0 & 0 & 0 & 0 \\
0 & 0 & 1 & -1 & 0 & 0 & 0 & 0 \\
0 & 0 & 0 & 0 & 1 & 1 & 0 & 0 \\
0 & 0 & 0 & 0 & 0 & 0 & 1 & -1
\end{array}\right)
$$


Furthermore, the matrix of integral and double integral of Haar wavelet with respect to the collocation grid are given as

$$
\mathcal{P}=\frac{1}{16}\left(\begin{array}{cccccccc}
1 & 3 & 5 & 7 & 9 & 11 & 13 & 15 \\
1 & 3 & 5 & 7 & 7 & 5 & 3 & 1 \\
1 & 3 & 3 & 1 & 0 & 0 & 0 & 0 \\
0 & 0 & 0 & 0 & 1 & 3 & 3 & 1 \\
1 & 1 & 0 & 0 & 0 & 0 & 0 & 0 \\
0 & 0 & 1 & 1 & 0 & 0 & 0 & 0 \\
0 & 0 & 0 & 0 & 1 & 1 & 0 & 0 \\
0 & 0 & 0 & 0 & 0 & 0 & 1 & 1
\end{array}\right), \quad \mathcal{Q}=\frac{1}{512}\left(\begin{array}{cccccccc}
1 & 9 & 25 & 49 & 81 & 121 & 169 & 225 \\
1 & 9 & 25 & 49 & 79 & 103 & 119 & 127 \\
1 & 9 & 23 & 31 & 32 & 32 & 32 & 32 \\
0 & 0 & 0 & 0 & 1 & 9 & 23 & 31 \\
1 & 7 & 8 & 8 & 8 & 8 & 8 & 8 \\
0 & 0 & 1 & 7 & 8 & 8 & 8 & 8 \\
0 & 0 & 0 & 0 & 1 & 7 & 8 & 8 \\
0 & 0 & 0 & 0 & 0 & 0 & 1 & 7
\end{array}\right)
$$

\section{Treatment of Singularly Perturbed Differential Equa- tions with Delay and Shift}

\subsection{Methods for Solving Linear Singularly Perturbed Differential Equa- tions with Delay and Shift using Taylor's Series}

In order to solve the singularly perturbed differential equation with delay and shift, we first convert the delay term $y(t-\delta)$ and shift term $y(t+\eta)$ using Taylor series expansion and then apply Haar wavelet method to solve the problem. By Taylor series expansion (upto first order), we obtain the following approximations for $y(t-\delta)$ and $y(t+\eta)$ as

$$
y(t-\delta) \approx y(t)-\delta y^{\prime}(t)
$$

and

$$
y(t+\eta) \approx y(t)+\eta y^{\prime}(t) .
$$

Using equation (3.1) and (3.2) in equation (1.1), we obtain

$$
\epsilon^{2} y^{\prime \prime}(t)+(c(t) \eta-a(t) \delta) y^{\prime}(t)+(b(t)+a(t)+c(t)) y(t)=f(t), \quad t \in(0,1)
$$

To solve this problem, we assume that

$$
y^{\prime \prime}(t)=\sum_{i=1}^{N} a_{i} \mathcal{H}_{i}(t)
$$

Now integrating from 0 to $t$, we get

$$
y^{\prime}(t)=\sum_{i=1}^{N} a_{i} \mathcal{P}_{i}(t)+y^{\prime}(0) .
$$

Further to find $y^{\prime}(0)$, integrate the equation (3.4) from 0 to 1 , we get

$$
y^{\prime}(0)=y(1)-y(0)-\sum_{i=1}^{N} a_{i} \mathcal{C}_{i}(t)
$$


where $\mathcal{C}_{i}(t)=\int_{0}^{1} \mathcal{P}_{i}(t) d t$

Again integrating equation (3.5) from 0 to $t$, we get

$$
y(t)=\sum_{i=1}^{N} a_{i} \mathcal{Q}_{i}(t)+t y^{\prime}(0)+y(0) .
$$

Now, using equation (3.6) in equations (3.5) and (3.7), we get

$$
y^{\prime}(t)=\sum_{i=1}^{N} a_{i} \mathcal{P} i(t)+y(1)-y(0)-\sum_{i=1}^{N} a_{i} \mathcal{C}_{i}(t),
$$

and

$$
y(t)=\sum_{i=1}^{N} a_{i} \mathcal{Q}_{i}(t)+t\left(y(1)-y(0)-\sum_{i=1}^{N} a_{i} \mathcal{C}_{i}(t)\right)+y(0) .
$$

Using equations (3.4), (3.8) and (3.9) in equation (3.3), we get the following system of linear equations:

$$
\begin{array}{r}
\epsilon^{2} \sum_{i=1}^{N} a_{i} \mathcal{H}_{i}(t)+(c(t) \eta-a(t) \delta)\left(\sum_{i=1}^{N} a_{i} \mathcal{P}_{i}(t)+y(1)-y(0)-\sum_{i=1}^{N} a_{i} \mathcal{C}_{i}(t)\right) \\
+(b(t)+a(t)+c(t))\left(\sum_{i=1}^{N} a_{i} \mathcal{Q}(t)+t\left(y(1)-y(0)-\sum_{i=1}^{N} a_{i} \mathcal{C}(t)\right)\right)=f(t) \\
\sum_{i=1}^{N} a_{i}\left(\epsilon^{2} \mathcal{H}_{i}(t)+(c(t) \eta-a(t) \delta)\left(\mathcal{P}_{i}(t)-\mathcal{C}_{i}(t)\right)+(b(t)+a(t)+c(t))(\mathcal{Q}(t)-t \mathcal{C}(t))\right) \\
=f(t)+(y(0)-y(1))(c(t) \eta-a(t) \delta+t(b(t)+a(t)+c(t))) .
\end{array}
$$

with the interval conditions (1.2) and (1.3). Now, we can easily find the Haar wavelet coefficient $a_{i}$ 's by solving this system of linear equations by using Gauss elimination method or any other method for linear equation and then put them in equation (3.9) in order to get Haar wavelet solution of singularly perturbed differential equation with delay and shift.

\subsection{Direct Methods for Solving Linear Singularly Perturbed Differen- tial Equations with Delay and Shift}

We apply Haar wavelet collocation method directly to solve the (1.1) with conditions (1.2) and (1.3). We replace $t$ by $t-\delta$ and $t+\eta$ respectively in equation (3.9) and then we obtain

$$
y(t-\delta)=\sum_{i=1}^{N} a_{i} \mathcal{Q}_{i}(t-\delta)+(t-\delta)\left(y(1)-y(0)-\sum_{i=1}^{N} a_{i} \mathcal{C}_{i}(t-\delta)\right)+y(0)
$$

and

$$
y(t+\eta)=\sum_{i=1}^{N} a_{i} \mathcal{Q}_{i}(t+\eta)+(t+\eta)\left(y(1)-y(0)-\sum_{i=1}^{N} a_{i} \mathcal{C}_{i}(t+\eta)\right)+y(0)
$$


On using equations (3.4), (3.9), (3.12) and (3.13) in the equation (1.1) we obtain the following system of linear equations:

$$
\begin{aligned}
& \epsilon^{2}\left(\sum_{i=1}^{N} a_{i} \mathcal{H}_{i}(t)\right)+a(t)\left(\sum_{i=1}^{N} a_{i} \mathcal{Q}_{i}(t-\delta)+(t-\delta)\left(y(1)-y(0)-\sum_{i=1}^{N} a_{i} \mathcal{C}_{i}(t-\delta)\right)+y(0)\right) \\
& +b(t)\left(\sum_{i=1}^{N} a_{i} \mathcal{Q}_{i}(t)+t\left(y(1)-y(0)-\sum_{i=1}^{N} a_{i} \mathcal{C}_{i}(t)\right)+y(0)\right)+c(t)\left(\sum_{i=1}^{N} a_{i} \mathcal{Q}_{i}(t+\eta)+(t+\eta)\right. \\
& \left.\left(y(1)-y(0)-\sum_{i=1}^{N} a_{i} \mathcal{C}_{i}(t+\eta)\right)+y(0)\right)=f(t) \\
& \quad \sum_{i=1}^{N} a_{i}\left(\epsilon^{2} \mathcal{H}_{i}(t)+a(t)\left(\mathcal{Q}_{i}(t-\delta)-(t-\delta) \mathcal{C}_{i}(t-\delta)\right)+b(t)\left(\mathcal{Q}_{i}(t)-t \mathcal{C}_{i}(t)\right)\right. \\
& \left.\quad+c(t)\left(\mathcal{Q}_{i}(t+\eta)-(t+\eta) \mathcal{C}_{i}(t+\eta)\right)\right)=f(t)-a(t)((t-\delta)(y(1)-y(0))+y(0)) \\
& \quad-b(t)(t(y(1)-y(0))+y(0))-c(t)((t+\eta)(y(1)-y(0))+y(0))
\end{aligned}
$$

with the interval conditions (1.2) and (1.3). Now, we can easily find the Haar wavelet coefficient $a_{i}$ 's by solving this system of linear equations by using Gauss elimination method or any other method for linear equation and then put them in equation (3.9) in order to get Haar wavelet solution of singularly perturbed differential equation with delay and shift directly without using Taylor series expansion.

\subsection{Error Analysis}

Lemma 3.1. Let $y(x)$ be a square integrable function with bounded first derivative on $(0,1)$ and $y\left(x_{j}\right)$ be its Haar wavelet approximation, then the error norm at $J^{\text {th }}$ level satisfies the inequality

$$
\|E\| \leq 2 D \sqrt{K}\left(\frac{2^{-2(J+1)}}{3}\right)^{2},
$$

where $K$ is a positive constant and $D$ is given by $y^{\prime}(t) \leq D$.

Proof. For the proof see $[9,27]$.

In case, if we do not have the exact solution of the singularly perturbed differential equations with delay and shift, then the maximum absolute residual error is calculated by the following formula :

$$
E=\max \left|\epsilon^{2} y_{J}^{\prime \prime}\left(t_{j}\right)+\left(c\left(t_{j}\right) \eta-a\left(t_{j}\right) \delta\right) y_{J}^{\prime}\left(t_{j}\right)+\left(b\left(t_{j}\right)+a\left(t_{j}\right)+c\left(t_{j}\right)\right) y_{J}\left(t_{j}\right)-f\left(t_{j}\right)\right|,
$$

where $y_{J}^{\prime \prime}\left(t_{j}\right), y_{J}^{\prime}\left(t_{j}\right)$ and $y_{J}\left(t_{j}\right)$ are given in equations $(3.4),(3.8)$ and $(3.9), j=1,2, \ldots N$, $N=2^{J+1}$.

In case of direct Haar wavelet method we computed the maximum absolute residual error by the following formula:

$$
E=\max \left|\epsilon^{2} y_{J}^{\prime \prime}\left(t_{j}\right)+a\left(t_{j}\right) y_{J}\left(t_{j}-\delta\right)+b\left(t_{j}\right) y_{J}\left(t_{j}\right)+c\left(t_{j}\right) y\left(t_{J}+\eta\right)-f\left(t_{j}\right)\right|,
$$


where $y_{J}^{\prime \prime}\left(t_{j}\right), y_{J}\left(t_{j}\right), y_{J}\left(t_{j}-\delta\right)$ and $y_{J}\left(t_{j}+\eta\right)$ are given in equations (3.4), (3.9), (3.12) and (3.13), $j=1,2, \ldots N, N=2^{J+1}$.

\section{$4 \quad$ Numerical Examples}

To illustrate the Haar wavelet method, in this section, we demonstrate numerical examples of some linear problems of singularly perturbed differential equations with delay and shift. The results are tabulated and also compared with the finite difference and fitted operator finite difference method presented in [11] and [29] respectively.

The exact solution for the problems 1,2 and 3 is given by

$$
y(t)=\frac{(a(t)+b(t)+c(t)-1)\left\{\left(e^{m_{2}}-1\right) e^{m_{1} t}-\left(e^{m_{1}}-1\right) e^{m_{2} t}\right\}}{(a(t)+b(t)+c(t))\left\{e^{m_{2}}-e^{m_{1}}\right\}}+\frac{1}{(a(t)+b(t)+c(t))}
$$

where,

$$
\begin{aligned}
& m_{1}=\frac{(a(t) \delta-b(t) \eta)+\sqrt{(b(t) \eta-a(t) \delta)-4\left(\epsilon^{2}(a(t)+b(t)+c(t))\right)}}{2 \epsilon^{2}} \\
& m_{2}=\frac{(a(t) \delta-b(t) \eta)-\sqrt{(b(t) \eta-a(t) \delta)-4\left(\epsilon^{2}(a(t)+b(t)+c(t))\right)}}{2 \epsilon^{2}}
\end{aligned}
$$

Problem 1. Let us consider the following SPDDE with no term containing positive shift

$$
\epsilon^{2} y^{\prime \prime}(t)-2 y(t-\delta)-y(t)=1, \quad t \in(0,1)
$$

with boundary conditions

$$
y(0)=1, \quad-\delta \leq t \leq 0, \quad y(1)=1 .
$$

Maximum absolute errors (MAE) for a particular value of perturbation parameter and various values of delay parameter obtained by Haar wavelet method with different resolutions level have been given in the tables 1.1 and 1.2. MAE obtained by direct Haar wavelet method is given in the table 1.3 and finite difference method [11] is given in the table 1.4. Also, graph of analytical and Haar wavelet solution is given in figures 1, 2 and 3.

Table 1.1 Maximum absolute errors obtained by Haar wavelet for different values of $\delta$ with $\epsilon=10^{-1}$.

\begin{tabular}{ccccc}
\hline $\mathrm{N}$ & $\delta=0.0$ & $\delta=0.3 \epsilon$ & $\delta=0.6 \epsilon$ & $\delta=0.9 \epsilon$ \\
\hline 32 & $8.4849 \mathrm{e}-05$ & $1.8594 \mathrm{e}-04$ & $3.4514 \mathrm{e}-04$ & $5.5944 \mathrm{e}-04$ \\
64 & $1.1444 \mathrm{e}-05$ & $2.4442 \mathrm{e}-05$ & $4.4929 \mathrm{e}-05$ & $7.4243 \mathrm{e}-05$ \\
128 & $1.4550 \mathrm{e}-06$ & $3.1050 \mathrm{e}-06$ & $5.6912 \mathrm{e}-06$ & $9.4117 \mathrm{e}-06$ \\
256 & $1.8248 \mathrm{e}-07$ & $3.8959 \mathrm{e}-07$ & $7.1394 \mathrm{e}-07$ & $1.1778 \mathrm{e}-06$ \\
512 & $2.2834 \mathrm{e}-08$ & $4.8731 \mathrm{e}-08$ & $8.9287 \mathrm{e}-08$ & $1.4750 \mathrm{e}-07$ \\
1024 & $2.8549 \mathrm{e}-09$ & $6.0932 \mathrm{e}-09$ & $1.1165 \mathrm{e}-08$ & $1.8445 \mathrm{e}-08$ \\
2048 & $3.5688 \mathrm{e}-10$ & $7.6169 \mathrm{e}-10$ & $1.3957 \mathrm{e}-09$ & $2.3058 \mathrm{e}-09$ \\
\hline
\end{tabular}


Table 1.2 Maximum absolute errors obtained by Haar wavelet for different values of $\delta$ with $\epsilon=10^{-2}$.

\begin{tabular}{ccccc}
\hline $\mathrm{N}$ & $\delta=0.0$ & $\delta=0.3 \epsilon$ & $\delta=0.6 \epsilon$ & $\delta=0.9 \epsilon$ \\
\hline 32 & $1.2000 \mathrm{e}-03$ & $2.1000 \mathrm{e}-03$ & $2.6000 \mathrm{e}-03$ & $4.8000 \mathrm{e}-03$ \\
64 & $2.9753 \mathrm{e}-04$ & $3.6274 \mathrm{e}-04$ & $1.1000 \mathrm{e}-03$ & $2.2000 \mathrm{e}-03$ \\
128 & $9.4676 \mathrm{e}-05$ & $1.7471 \mathrm{e}-04$ & $2.9650 \mathrm{e}-04$ & $5.5434 \mathrm{e}-04$ \\
256 & $1.6234 \mathrm{e}-05$ & $3.5419 \mathrm{e}-05$ & $6.3325 \mathrm{e}-05$ & $9.8313 \mathrm{e}-05$ \\
512 & $2.1993 \mathrm{e}-06$ & $4.7520 \mathrm{e}-06$ & $8.6386 \mathrm{e}-06$ & $1.4088 \mathrm{e}-05$ \\
1024 & $2.8249 \mathrm{e}-07$ & $6.0290 \mathrm{e}-07$ & $1.1082 \mathrm{e}-06$ & $1.8335 \mathrm{e}-06$ \\
2048 & $3.5608 \mathrm{e}-08$ & $7.5960 \mathrm{e}-08$ & $1.3937 \mathrm{e}-07$ & $2.3009 \mathrm{e}-07$ \\
\hline
\end{tabular}

Table 1.3 Maximum absolute errors obtained by direct Haar wavelet method for different values of $\delta$ with $\epsilon=10^{-2}$.

\begin{tabular}{ccccc}
\hline $\mathrm{N}$ & $\delta=0.0$ & $\delta=0.3 \epsilon$ & $\delta=0.6 \epsilon$ & $\delta=0.9 \epsilon$ \\
\hline 32 & $1.200 \mathrm{e}-03$ & $1.3000 \mathrm{e}-03$ & $1.4000 \mathrm{e}-03$ & $1.500 \mathrm{e}-03$ \\
64 & $2.9753 \mathrm{e}-04$ & $3.0878 \mathrm{e}-04$ & $3.2049 \mathrm{e}-04$ & $3.3238 \mathrm{e}-04$ \\
128 & $9.4676 \mathrm{e}-05$ & $1.0333 \mathrm{e}-04$ & $1.1480 \mathrm{e}-04$ & $1.2907 \mathrm{e}-04$ \\
256 & $1.6234 \mathrm{e}-05$ & $1.8660 \mathrm{e}-05$ & $2.3024 \mathrm{e}-05$ & $2.9441 \mathrm{e}-05$ \\
512 & $2.1993 \mathrm{e}-06$ & $2.8222 \mathrm{e}-06$ & $4.3724 \mathrm{e}-06$ & $6.9155 \mathrm{e}-06$ \\
\hline
\end{tabular}

Table 1.4 Maximum absolute errors obtained in [11] for different values of $\delta$ and $\epsilon$.

\begin{tabular}{cccccc}
\hline$\epsilon$ & $\mathrm{N}$ & $10^{2}$ & $10^{3}$ & $10^{4}$ & $10^{5}$ \\
\hline$\epsilon=0.1$ & $\delta=0.0$ & $6.1101 \mathrm{e}-04$ & $6.1300 \mathrm{e}-06$ & $6.0000 \mathrm{e}-08$ & 0.00000000 \\
& $\delta=0.3 \epsilon$ & $8.8888 \mathrm{e}-04$ & $8.6383 \mathrm{e}-04$ & $8.6130 \mathrm{e}-05$ & $8.6130 \mathrm{e}-06$ \\
& $\delta=0.6 \epsilon$ & $1.8934 \mathrm{e}-02$ & $1.9461 \mathrm{e}-03$ & $1.9519 \mathrm{e}-04$ & $1.9519 \mathrm{e}-05$ \\
& $\delta=0.9 \epsilon$ & $3.0057 \mathrm{e}-02$ & $3.1753 \mathrm{e}-03$ & $3.1941 \mathrm{e}-04$ & $3.1941 \mathrm{e}-05$ \\
\hline$\epsilon=0.01$ & $\delta=0.0$ & $4.2387 \mathrm{e}-02$ & $5.0956 \mathrm{e}-04$ & $5.1100 \mathrm{e}-06$ & $5.0000 \mathrm{e}-08$ \\
& $\delta=0.3 \epsilon$ & $8.1361 \mathrm{e}-02$ & $6.4128 \mathrm{e}-03$ & $6.1801 \mathrm{e}-04$ & $6.1570 \mathrm{e}-05$ \\
& $\delta=0.6 \epsilon$ & $1.1198 \mathrm{e}-01$ & $1.1306 \mathrm{e}-02$ & $1.1333 \mathrm{e}-03$ & $1.1338 \mathrm{e}-04$ \\
& $\delta=0.9 \epsilon$ & $1.3267 \mathrm{e}-01$ & $1.4300 \mathrm{e}-02$ & $1.4420 \mathrm{e}-03$ & $1.4433 \mathrm{e}-04$ \\
\hline
\end{tabular}

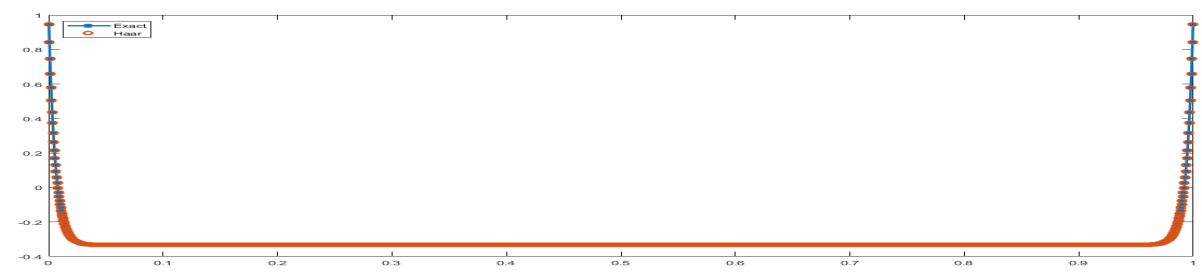

Figure 1. Exact and Haar wavelet solution of problem 1 for $\epsilon=0.01$ and $\delta=0.0$ with $J=10$. 


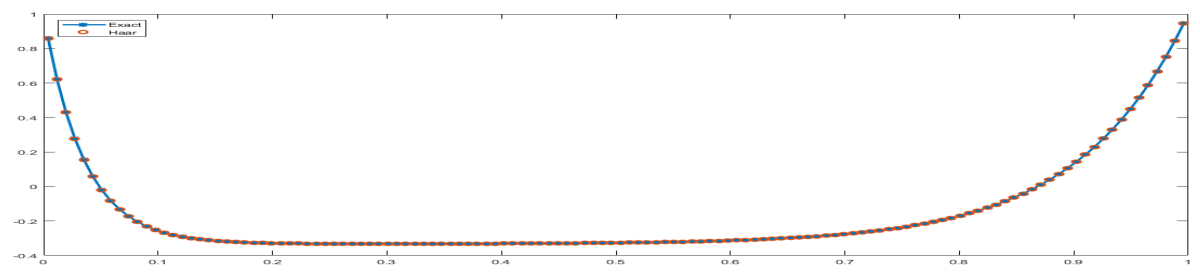

Figure 2. Exact and Haar wavelet solution of problem 1 for $\epsilon=0.1$ and $\delta=0.9 \epsilon$ with $J=6$.

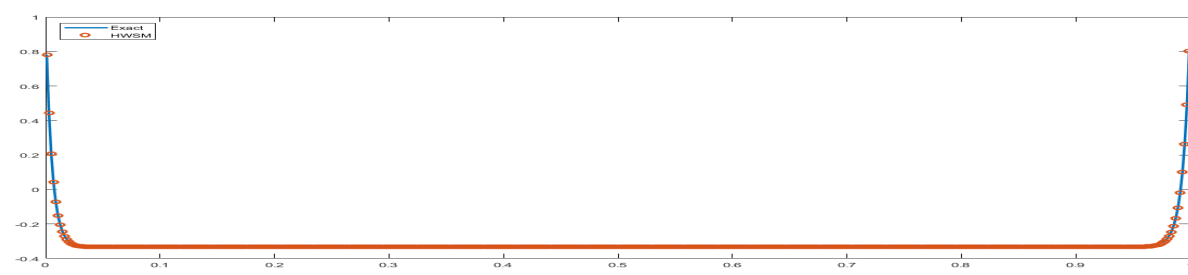

Figure 3. Exact and direct Haar wavelet solution of problem 1 for $\epsilon=0.01$ and $\delta=0.9 \epsilon$ with $J=8$.

Problem 2. Let us consider the following SPDDE with no term containing negative shift

$$
\epsilon^{2} y^{\prime \prime}(t)-y(t)-2 y(t+\eta)=1, \quad t \in(0,1)
$$

with boundary conditions

$$
y(0)=1, \quad y(1)=1, \quad 1 \leq t \leq 1+\eta
$$

Maximum absolute errors (MAE) for a particular value of perturbation parameter and various values of shift parameter obtained by Haar wavelet method with different resolutions level have been given in the tables 2.1 and 2.2. MAE obtained by direct Haar wavelet method is given in the table 2.3 and finite difference method [11] is given in the table 2.4. Also, graph of analytical and Haar wavelet solution is given in figures 4,5 and 6 .

Table 2.1 Maximum absolute errors obtained by Haar wavelet for different values of $\eta$ with $\epsilon=10^{-1}$.

\begin{tabular}{ccccc}
\hline $\mathrm{N}$ & $\eta=0.0$ & $\eta=0.3 \epsilon$ & $\eta=0.6 \epsilon$ & $\eta=0.9 \epsilon$ \\
\hline 32 & $8.4849 \mathrm{e}-05$ & $1.8594 \mathrm{e}-04$ & $3.4514 \mathrm{e}-04$ & $5.5944 \mathrm{e}-04$ \\
64 & $1.1444 \mathrm{e}-05$ & $2.4442 \mathrm{e}-05$ & $4.4929 \mathrm{e}-05$ & $7.4243 \mathrm{e}-05$ \\
128 & $1.4550 \mathrm{e}-06$ & $3.1050 \mathrm{e}-06$ & $5.6912 \mathrm{e}-06$ & $9.4117 \mathrm{e}-06$ \\
256 & $1.8248 \mathrm{e}-07$ & $3.8959 \mathrm{e}-07$ & $7.1394 \mathrm{e}-07$ & $1.1778 \mathrm{e}-06$ \\
512 & $2.2834 \mathrm{e}-08$ & $4.8731 \mathrm{e}-08$ & $8.9287 \mathrm{e}-08$ & $1.4750 \mathrm{e}-07$ \\
1024 & $2.8549 \mathrm{e}-09$ & $6.0932 \mathrm{e}-09$ & $1.1165 \mathrm{e}-08$ & $1.8445 \mathrm{e}-08$ \\
2048 & $3.5688 \mathrm{e}-10$ & $7.6169 \mathrm{e}-10$ & $1.3957 \mathrm{e}-09$ & $2.3058 \mathrm{e}-09$ \\
\hline
\end{tabular}


Table 2.2 Maximum absolute errors obtained by Haar wavelet for different values of $\eta$ with $\epsilon=10^{-2}$.

\begin{tabular}{ccccc}
\hline $\mathrm{N}$ & $\eta=0.0$ & $\eta=0.3 \epsilon$ & $\eta=0.6 \epsilon$ & $\eta=0.9 \epsilon$ \\
\hline 32 & $1.200 \mathrm{e}-03$ & $2.1000 \mathrm{e}-03$ & $2.6000 \mathrm{e}-03$ & $1.1000 \mathrm{e}-03$ \\
64 & $2.9753 \mathrm{e}-04$ & $3.6274 \mathrm{e}-04$ & $1.100 \mathrm{e}-03$ & $2.2000 \mathrm{e}-03$ \\
128 & $9.4676 \mathrm{e}-05$ & $1.7471 \mathrm{e}-04$ & $2.9650 \mathrm{e}-04$ & $5.5434 \mathrm{e}-04$ \\
256 & $1.6234 \mathrm{e}-05$ & $3.5419 \mathrm{e}-05$ & $6.3325 \mathrm{e}-05$ & $9.8313 \mathrm{e}-05$ \\
512 & $2.1993 \mathrm{e}-06$ & $4.7520 \mathrm{e}-06$ & $8.6386 \mathrm{e}-06$ & $1.4088 \mathrm{e}-05$ \\
1024 & $2.8249 \mathrm{e}-07$ & $6.0290 \mathrm{e}-07$ & $1.1082 \mathrm{e}-06$ & $1.8335 \mathrm{e}-06$ \\
2048 & $3.5608 \mathrm{e}-08$ & $7.5960 \mathrm{e}-08$ & $1.3937 \mathrm{e}-07$ & $2.3009 \mathrm{e}-07$ \\
\hline
\end{tabular}

Table 2.3 Maximum absolute errors obtained by direct Haar wavelet method for different values of $\eta$ with $\epsilon=10^{-2}$.

\begin{tabular}{ccccc}
\hline $\mathrm{N}$ & $\eta=0.0$ & $\eta=0.3 \epsilon$ & $\eta=0.6 \epsilon$ & $\eta=0.9 \epsilon$ \\
\hline 32 & $1.200 \mathrm{e}-03$ & $1.3000 \mathrm{e}-03$ & $1.4000 \mathrm{e}-03$ & $1.5000 \mathrm{e}-03$ \\
64 & $2.9753 \mathrm{e}-04$ & $3.0878 \mathrm{e}-04$ & $3.2049 \mathrm{e}-04$ & $3.3238 \mathrm{e}-04$ \\
128 & $9.4676 \mathrm{e}-05$ & $1.0333 \mathrm{e}-04$ & $1.1480 \mathrm{e}-04$ & $1.2907 \mathrm{e}-04$ \\
256 & $1.6234 \mathrm{e}-05$ & $1.8660 \mathrm{e}-05$ & $2.3024 \mathrm{e}-05$ & $2.9441 \mathrm{e}-05$ \\
512 & $2.1993 \mathrm{e}-06$ & $2.8222 \mathrm{e}-06$ & $4.3724 \mathrm{e}-06$ & $6.9155 \mathrm{e}-06$ \\
\hline
\end{tabular}

Table 2.4 Maximum absolute errors obtained in [11] for different values of $\eta$ and $\epsilon$.

\begin{tabular}{cccccc}
\hline$\epsilon$ & $\mathrm{N}$ & $10^{2}$ & $10^{3}$ & $10^{4}$ & $10^{5}$ \\
\hline$\epsilon=0.1$ & $\delta=0.0$ & $6.1101 \mathrm{e}-04$ & $6.1300 \mathrm{e}-06$ & $6.000 \mathrm{e}-08$ & 0.0000 \\
& $\delta=0.3 \epsilon$ & $5.50887 \mathrm{e}-03$ & $6.04300 \mathrm{e}-04$ & $6.0950 \mathrm{e}-05$ & $6.100 \mathrm{e}-06$ \\
& $\delta=0.6 \epsilon$ & $9.48956 \mathrm{e}-03$ & $9.85910 \mathrm{e}-04$ & $9.8940 \mathrm{e}-05$ & $9.900 \mathrm{e}-06$ \\
& $\delta=0.9 \epsilon$ & $1.167763 \mathrm{e}-02$ & $1.18605 \mathrm{e}-03$ & $1.1878 \mathrm{e}-04$ & $1.1880 \mathrm{e}-05$ \\
\hline$\epsilon=0.01$ & & & & & \\
& $\delta=0.0$ & $4.2387 \mathrm{e}-02$ & $5.0956 \mathrm{e}-04$ & $5.1100 \mathrm{e}-06$ & $5.0000 \mathrm{e}-08$ \\
& $\delta=0.3 \epsilon$ & $1.1620 \mathrm{e}-02$ & $5.0693 \mathrm{e}-03$ & $5.573 \mathrm{e}-04$ & $5.6220 \mathrm{e}-05$ \\
& $\delta=0.6 \epsilon$ & $4.6564 \mathrm{e}-02$ & $9.2472 \mathrm{e}-03$ & $9.6281 \mathrm{e}-04$ & $9.6640 \mathrm{e}-05$ \\
& $\delta=0.9 \epsilon$ & $9.6921 \mathrm{e}-02$ & $1.1664 \mathrm{e}-02$ & $1.1860 \mathrm{e}-03$ & $1.1878 \mathrm{e}-04$ \\
\hline
\end{tabular}

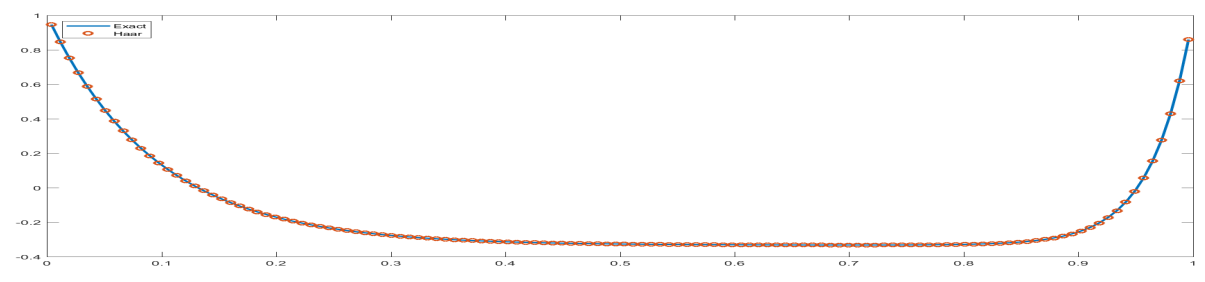

Figure 4. Exact and Haar wavelet solution of problem 2 for $\epsilon=0.1$ and $\eta=0.9 \epsilon$ with $J=6$. 


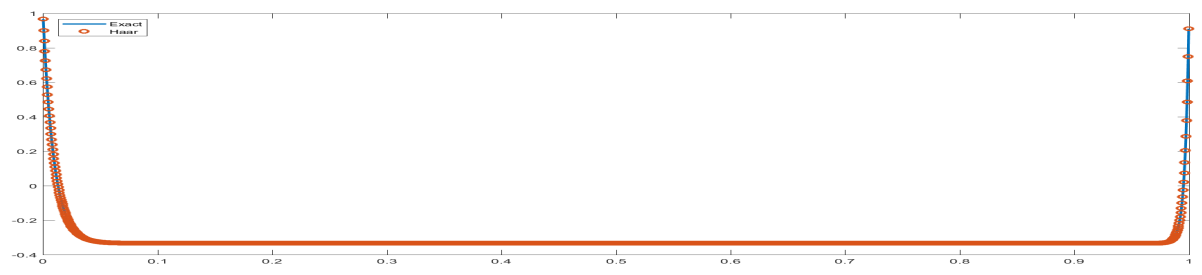

Figure 5. Exact and Haar wavelet solution of problem 2 for $\epsilon=0.01$ and $\eta=0.9 \epsilon$ with $J=10$.

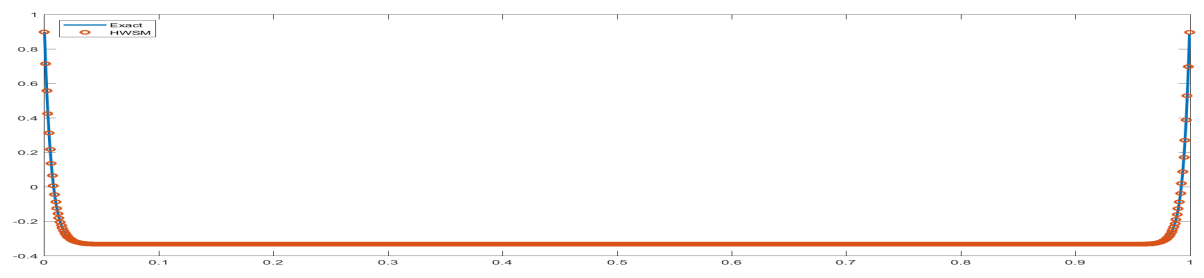

Figure 6. Exact and direct Haar wavelet method solution of problem 2 for $\epsilon=0.01$ and $\eta=0.9 \epsilon$ with $J=9$.

Problem 3. Let us consider the following SPDDE with term containing both delay and shift

$$
\epsilon^{2} y^{\prime \prime}(t)+\frac{1}{4} y(t-\delta)-y(t)+\frac{1}{4} y(t+\eta)=1, \quad t \in(0,1)
$$

with boundary conditions

$$
y(0)=1, \text { on } \quad-\delta \leq t \leq 0, \quad y(1)=1, \quad 1 \leq t \leq 1+\eta
$$

Maximum absolute errors (MAE) for a particular value of perturbation parameter and various values of delay and shift parameters obtained by Haar wavelet method with different resolutions level have been given in the tables 3.1, 3.2, 3.3 and 3.4. MAE obtained by direct Haar wavelet method is given in the table 3.5 and finite difference method [11] have been given in the tables 3.6 and 3.7. Also, graph of analytical and Haar wavelet solution is given in figures 7, 8 and 9 .

Table 3.1 Maximum absolute errors obtained by Haar wavelet for different values of $\eta$ with $\epsilon=10^{-1}$ and $\delta=0.07$.

\begin{tabular}{ccccc}
\hline $\mathrm{N}$ & $\eta=0.0$ & $\eta=0.3 \epsilon$ & $\eta=0.6 \epsilon$ & $\eta=0.9 \epsilon$ \\
\hline 32 & $6.1312 \mathrm{e}-05$ & $4.9352 \mathrm{e}-05$ & $3.8893 \mathrm{e}-05$ & $4.2215 \mathrm{e}-05$ \\
64 & $7.7492 \mathrm{e}-06$ & $6.2204 \mathrm{e}-06$ & $4.9043 \mathrm{e}-06$ & $5.3151 \mathrm{e}-06$ \\
128 & $9.7134 \mathrm{e}-07$ & $7.7923 \mathrm{e}-07$ & $6.1454 \mathrm{e}-07$ & $6.6635 \mathrm{e}-07$ \\
256 & $1.2149 \mathrm{e}-07$ & $9.7461 \mathrm{e}-08$ & $7.6856 \mathrm{e}-08$ & $8.3358 \mathrm{e}-08$ \\
512 & $1.5188 \mathrm{e}-08$ & $1.2184 \mathrm{e}-08$ & $9.6086 \mathrm{e}-09$ & $1.0422 \mathrm{e}-08$ \\
1024 & $1.8986 \mathrm{e}-09$ & $1.5231 \mathrm{e}-09$ & $1.2011 \mathrm{e}-09$ & $1.3028 \mathrm{e}-09$ \\
2048 & $2.3733 \mathrm{e}-10$ & $1.9039 \mathrm{e}-10$ & $1.5014 \mathrm{e}-10$ & $1.6285 \mathrm{e}-10$ \\
\hline
\end{tabular}


Table 3.2 Maximum absolute errors obtained by Haar wavelet for different values of $\eta$ with $\epsilon=10^{-2}$ and $\delta=0.07$.

\begin{tabular}{ccccc}
\hline $\mathrm{N}$ & $\eta=0.0$ & $\eta=0.3 \epsilon$ & $\eta=0.6 \epsilon$ & $\eta=0.9 \epsilon$ \\
\hline 32 & $3.8800 \mathrm{e}-02$ & $3.600 \mathrm{e}-02$ & $3.3300 \mathrm{e}-02$ & $3.0700 \mathrm{e}-02$ \\
64 & $8.800 \mathrm{e}-03$ & $8.1000 \mathrm{e}-03$ & $7.5000 \mathrm{e}-04$ & $6.800 \mathrm{e}-03$ \\
128 & $1.200 \mathrm{e}-03$ & $1.1000 \mathrm{e}-03$ & $9.8687 \mathrm{e}-04$ & $8.9191 \mathrm{e}-04$ \\
256 & $1.7996 \mathrm{e}-04$ & $1.6663 \mathrm{e}-04$ & $1.5364 \mathrm{e}-04$ & $1.4105 \mathrm{e}-04$ \\
512 & $2.2778 \mathrm{e}-05$ & $2.0988 \mathrm{e}-05$ & $1.9259 \mathrm{e}-05$ & $1.7596 \mathrm{e}-05$ \\
1024 & $2.8467 \mathrm{e}-06$ & $2.6326 \mathrm{e}-06$ & $2.4245 \mathrm{e}-06$ & $2.2231 \mathrm{e}-06$ \\
2048 & $3.5674 \mathrm{e}-07$ & $3.2941 \mathrm{e}-07$ & $3.0292 \mathrm{e}-07$ & $2.7817 \mathrm{e}-07$ \\
\hline
\end{tabular}

Table 3.3 Maximum absolute errors obtained by Haar wavelet for different values of $\delta$ and $\eta=0.5 \epsilon$ with $\epsilon=10^{-1}$.

\begin{tabular}{ccccc}
\hline $\mathrm{N}$ & $\delta=0.0$ & $\delta=0.3 \epsilon$ & $\delta=0.6 \epsilon$ & $\delta=0.9 \epsilon$ \\
\hline 32 & $5.3171 \mathrm{e}-05$ & $4.2215 \mathrm{e}-05$ & $3.8893 \mathrm{e}-05$ & $4.9352 \mathrm{e}-05$ \\
64 & $6.7077 \mathrm{e}-06$ & $5.3151 \mathrm{e}-06$ & $4.9043 \mathrm{e}-06$ & $6.2204 \mathrm{e}-06$ \\
128 & $8.3991 \mathrm{e}-07$ & $6.6635 \mathrm{e}-07$ & $6.1454 \mathrm{e}-07$ & $7.7923 \mathrm{e}-07$ \\
256 & $1.0507 \mathrm{e}-07$ & $8.3358 \mathrm{e}-08$ & $7.6856 \mathrm{e}-08$ & $9.7461 \mathrm{e}-08$ \\
512 & $1.3136 \mathrm{e}-08$ & $1.0422 \mathrm{e}-08$ & $9.6086 \mathrm{e}-09$ & $1.2184 \mathrm{e}-08$ \\
1024 & $1.6421 \mathrm{e}-09$ & $1.3028 \mathrm{e}-09$ & $1.2011 \mathrm{e}-09$ & $1.5231 \mathrm{e}-09$ \\
2048 & $2.0526 \mathrm{e}-10$ & $1.6285 \mathrm{e}-10$ & $1.5014 \mathrm{e}-10$ & $1.9039 \mathrm{e}-10$ \\
\hline
\end{tabular}

Table 3.4 Maximum absolute errors obtained by Haar wavelet for different values of $\delta$ and $\eta=0.5 \epsilon$ with $\epsilon=10^{-2}$.

\begin{tabular}{ccccc}
\hline $\mathrm{N}$ & $\delta=0.0$ & $\delta=0.3 \epsilon$ & $\delta=0.6 \epsilon$ & $\delta=0.9 \epsilon$ \\
\hline 32 & $1.600 \mathrm{e}-03$ & $1.4000 \mathrm{e}-03$ & $1.300 \mathrm{e}-03$ & $1.5000 \mathrm{e}-03$ \\
64 & $4.8309 \mathrm{e}-04$ & $3.8851 \mathrm{e}-04$ & $3.5899 \mathrm{e}-04$ & $4.5059 \mathrm{e}-04$ \\
128 & $7.5563 \mathrm{e}-05$ & $5.8459 \mathrm{e}-05$ & $5.3565 \mathrm{e}-05$ & $6.9553 \mathrm{e}-05$ \\
256 & $1.0149 \mathrm{e}-05$ & $7.9826 \mathrm{e}-06$ & $7.3293 \mathrm{e}-06$ & $9.3920 \mathrm{e}-06$ \\
512 & $1.2857 \mathrm{e}-06$ & $1.0099 \mathrm{e}-06$ & $9.2898 \mathrm{e}-07$ & $1.1888 \mathrm{e}-06$ \\
1024 & $1.6128 \mathrm{e}-07$ & $1.2686 \mathrm{e}-07$ & $1.1665 \mathrm{e}-07$ & $1.4924 \mathrm{e}-07$ \\
2048 & $2.0180 \mathrm{e}-08$ & $1.5879 \mathrm{e}-08$ & $1.4596 \mathrm{e}-08$ & $1.8668 \mathrm{e}-08$ \\
\hline
\end{tabular}


Table 3.5 Maximum absolute errors obtained by direct Haar wavelet method for different values of $\delta$ and $\eta=0.5 \epsilon$ with $\epsilon=10^{-2}$.

\begin{tabular}{ccccc}
\hline $\mathrm{N}$ & $\delta=0.0$ & $\delta=0.3 \epsilon$ & $\delta=0.6 \epsilon$ & $\delta=0.9 \epsilon$ \\
\hline 32 & $1.300 \mathrm{e}-03$ & $1.3000 \mathrm{e}-03$ & $1.300 \mathrm{e}-03$ & $1.3000 \mathrm{e}-03$ \\
64 & $3.4223 \mathrm{e}-04$ & $3.3289 \mathrm{e}-04$ & $3.2750 \mathrm{e}-04$ & $3.3164 \mathrm{e}-04$ \\
128 & $5.0135 \mathrm{e}-05$ & $4.8415 \mathrm{e}-05$ & $4.6612 \mathrm{e}-05$ & $4.5539 \mathrm{e}-05$ \\
256 & $6.3338 \mathrm{e}-06$ & $5.9092 \mathrm{e}-06$ & $5.1198 \mathrm{e}-06$ & $4.0707 \mathrm{e}-06$ \\
512 & $5.5035 \mathrm{e}-07$ & $4.0754 \mathrm{e}-07$ & $3.6762 \mathrm{e}-08$ & $5.9512 \mathrm{e}-07$ \\
\hline
\end{tabular}

Table 3.6 Maximum absolute errors obtained in [11] for different values of $\eta$ with

$$
\delta=0.7 \epsilon \text { and } \epsilon=10^{-2} \text {. }
$$

\begin{tabular}{ccccc}
\hline $\mathrm{N}$ & $10^{2}$ & $10^{3}$ & $10^{4}$ & $10^{5}$ \\
\hline$\eta=0.0$ & $2.4735 \mathrm{e}-02$ & $3.8970 \mathrm{e}-03$ & $4.1008 \mathrm{e}-04$ & $4.1210 \mathrm{e}-05$ \\
$\eta=0.3 \epsilon$ & $6.0820 \mathrm{e}-03$ & $2.2315 \mathrm{e}-03$ & $2.4367 \mathrm{e}-04$ & $2.4570 \mathrm{e}-05$ \\
$\eta=0.6 \epsilon$ & $1.4937 \mathrm{e}-02$ & $4.1870 \mathrm{e}-04$ & $6.1680 \mathrm{e}-05$ & $6.3700 \mathrm{e}-06$ \\
$\eta=0.9 \epsilon$ & $3.3097 \mathrm{e}-02$ & $1.5756 \mathrm{e}-03$ & $1.3880 \mathrm{e}-04$ & $1.3690 \mathrm{e}-05$ \\
\hline
\end{tabular}

Table 3.7 Maximum absolute errors obtained in [11] for different values of $\delta$ with $\eta=0.5 \epsilon$ and $\epsilon=10^{-2}$.

\begin{tabular}{ccccc}
\hline $\mathrm{N}$ & $10^{2}$ & $10^{3}$ & $10^{4}$ & $10^{5}$ \\
\hline$\delta=0.0$ & $1.2268 \mathrm{e}-02$ & $6.2876 \mathrm{e}-03$ & $6.9355 \mathrm{e}-04$ & $6.9980 \mathrm{e}-05$ \\
$\delta=0.3 \epsilon$ & $2.9136 \mathrm{e}-02$ & $2.1996 \mathrm{e}-03$ & $2.8233 \mathrm{e}-04$ & $2.8850 \mathrm{e}-05$ \\
$\delta=0.6 \epsilon$ & $5.4924 \mathrm{e}-02$ & $1.9429 \mathrm{e}-04$ & $1.4674 \mathrm{e}-05$ & $1.4200 \mathrm{e}-05$ \\
$\delta=0.9 \epsilon$ & $7.5716 \mathrm{e}-02$ & $5.5171 \mathrm{e}-03$ & $5.2442 \mathrm{e}-04$ & $5.2170 \mathrm{e}-05$ \\
\hline
\end{tabular}

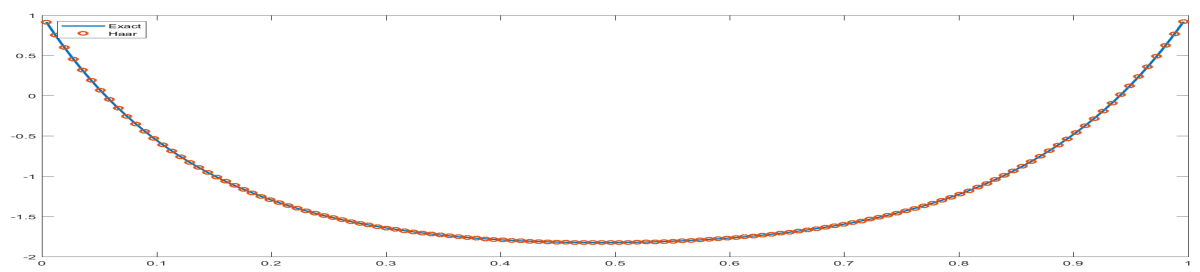

Figure 7. Exact and Haar wavelet solution of problem 3 for $\epsilon=0.1$ and $\delta=0.07$ with $J=6$.

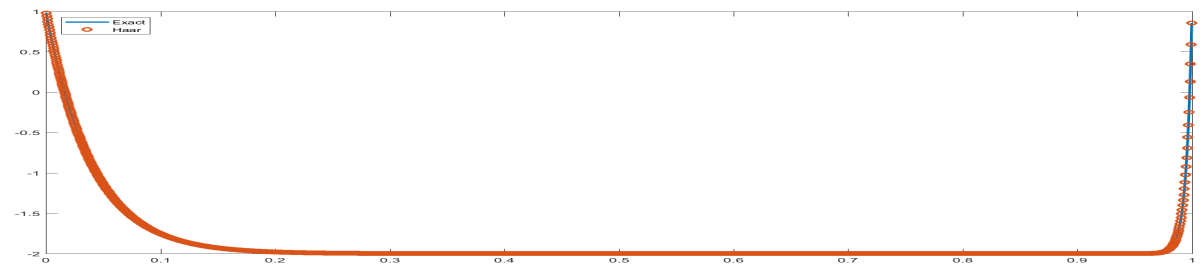


Figure 8. Exact and Haar wavelet solution of problem 3 for $\epsilon=0.01$ and $\eta=0.0$ with $J=10$.

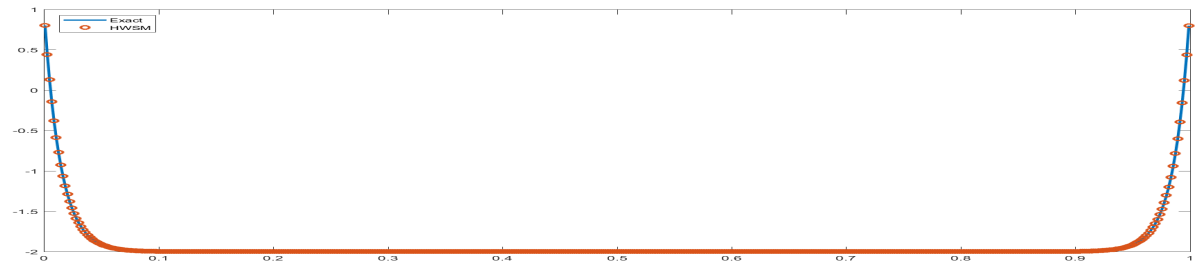

Figure 9. Exact and direct Haar wavelet solution of problem 3 for $\epsilon=0.01, \eta=0.5 \epsilon$ and $\delta=0.09 \epsilon$ with $J=8$.

Problem 4. Let us consider the following SPDDE with term containing both delay and shift

$$
\epsilon^{2} y^{\prime \prime}(t)-e^{t} y(t-\delta)+y(t)-(1+t) y(t+\eta)=1, \quad t \in(0,1)
$$

with boundary conditions

$$
y(0)=1, \quad-\delta \leq t \leq 0, \quad y(1)=1, \quad 1 \leq t \leq 1+\eta .
$$

The exact solution is not known.

Maximum absolute residual errors (MARE) for a particular value of perturbation parameter and various values of delay and shift parameters obtained by Haar wavelet method with different resolutions level is given in the table 4.1. MARE obtained by direct Haar wavelet method is given in the table 4.2 and non standard finite difference method [29] is given in the tables 4.3. Also, graph of Haar wavelet solution is given in figures 10 and 11.

Table 4.1 Maximum absolute residual errors obtained by Haar wavelet for fixed values of $\delta=0.5 \epsilon, \eta=0.5 \epsilon$ and different values of $\epsilon$.

\begin{tabular}{cccccccc}
\hline$\epsilon \backslash N$ & 32 & 64 & 128 & 256 & 512 & 1024 & 2048 \\
\hline $5^{-1}$ & $4.9960 \mathrm{e}-16$ & $3.3307 \mathrm{e}-16$ & $2.4633 \mathrm{e}-16$ & $1.9602 \mathrm{e}-16$ & $2.8276 \mathrm{e}-16$ & $1.4225 \mathrm{e}-16$ & $1.6827 \mathrm{e}-16$ \\
$5^{-2}$ & $8.6042 \mathrm{e}-16$ & $9.9226 \mathrm{e}-16$ & $9.6798 \mathrm{e}-16$ & $8.9512 \mathrm{e}-16$ & $9.2981 \mathrm{e}-16$ & $1.3422 \mathrm{e}-15$ & $1.0814 \mathrm{e}-15$ \\
$5^{-3}$ & $1.8874 \mathrm{e}-15$ & $4.3021 \mathrm{e}-15$ & $3.3237 \mathrm{e}-15$ & $7.3205 \mathrm{e}-15$ & $6.7325 \mathrm{e}-15$ & $1.0701 \mathrm{e}-14$ & $1.5781 \mathrm{e}-14$ \\
$5^{-4}$ & $1.3461 \mathrm{e}-15$ & $3.5874 \mathrm{e}-15$ & $8.1844 \mathrm{e}-15$ & $1.2448 \mathrm{e}-14$ & $3.3612 \mathrm{e}-14$ & $2.8293 \mathrm{e}-14$ & $4.3039 \mathrm{e}-14$ \\
$10^{-5}$ & $3.1225 \mathrm{e}-15$ & $2.9907 \mathrm{e}-15$ & $7.1818 \mathrm{e}-15$ & $2.4638 \mathrm{e}-14$ & $4.3722 \mathrm{e}-14$ & $1.0756 \mathrm{e}-13$ & $2.1428 \mathrm{e}-13$ \\
$10^{-6}$ & $1.3184 \mathrm{e}-15$ & $3.8164 \mathrm{e}-15$ & $7.5322 \mathrm{e}-15$ & $1.3583 \mathrm{e}-14$ & $4.3261 \mathrm{e}-14$ & $1.0861 \mathrm{e}-13$ & $2.2738 \mathrm{e}-13$ \\
$10^{-7}$ & $2.0262 \mathrm{e}-15$ & $4.9127 \mathrm{e}-15$ & $5.4401 \mathrm{e}-15$ & $1.3583 \mathrm{e}-14$ & $4.2241 \mathrm{e}-14$ & $1.0261 \mathrm{e}-13$ & $2.2161 \mathrm{e}-13$ \\
$10^{-8}$ & $1.6931 \mathrm{e}-15$ & $3.4486 \mathrm{e}-15$ & $1.0818 \mathrm{e}-14$ & $1.6629 \mathrm{e}-14$ & $3.8729 \mathrm{e}-14$ & $1.0039 \mathrm{e}-13$ & $2.1790 \mathrm{e}-13$ \\
$10^{-9}$ & $1.8457 \mathrm{e}-15$ & $2.9907 \mathrm{e}-15$ & $6.1860 \mathrm{e}-15$ & $1.1059 \mathrm{e}-14$ & $4.3895 \mathrm{e}-14$ & $9.6639 \mathrm{e}-14$ & $2.2028 \mathrm{e}-13$ \\
$10^{-10}$ & $2.4980 \mathrm{e}-15$ & $4.3923 \mathrm{e}-15$ & $7.7056 \mathrm{e}-15$ & $1.4043 \mathrm{e}-14$ & $4.8365 \mathrm{e}-14$ & $9.5973 \mathrm{e}-14$ & $2.1317 \mathrm{e}-13$ \\
$10^{-11}$ & $2.6784 \mathrm{e}-15$ & $2.9907 \mathrm{e}-15$ & $5.2458 \mathrm{e}-15$ & $1.4495 \mathrm{e}-14$ & $4.5006 \mathrm{e}-14$ & $9.9502 \mathrm{e}-14$ & $2.1895 \mathrm{e}-13$ \\
$10^{-12}$ & $1.8319 \mathrm{e}-15$ & $3.6846 \mathrm{e}-15$ & $9.8498 \mathrm{e}-15$ & $1.3385 \mathrm{e}-14$ & $4.1231 \mathrm{e}-14$ & $9.7948 \mathrm{e}-14$ & $2.2145 \mathrm{e}-13$ \\
$10^{-13}$ & $2.0262 \mathrm{e}-15$ & $2.9837 \mathrm{e}-15$ & $6.4254 \mathrm{e}-15$ & $1.2997 \mathrm{e}-14$ & $4.2119 \mathrm{e}-14$ & $1.0061 \mathrm{e}-13$ & $2.1718 \mathrm{e}-13$ \\
$10^{-14}$ & $1.6792 \mathrm{e}-15$ & $4.5797 \mathrm{e}-15$ & $6.3109 \mathrm{e}-15$ & $1.7382 \mathrm{e}-14$ & $4.4784 \mathrm{e}-14$ & $9.5644 \mathrm{e}-14$ & $2.1207 \mathrm{e}-13$ \\
$10^{-15}$ & $2.6784 \mathrm{e}-15$ & $3.3237 \mathrm{e}-15$ & $7.8861 \mathrm{e}-15$ & $1.4051 \mathrm{e}-14$ & $4.3250 \mathrm{e}-14$ & $9.5734 \mathrm{e}-14$ & $2.1961 \mathrm{e}-13$ \\
$10^{-16}$ & $3.1086 \mathrm{e}-15$ & $2.8449 \mathrm{e}-15$ & $1.1734 \mathrm{e}-14$ & $1.2443 \mathrm{e}-14$ & $4.1030 \mathrm{e}-14$ & $9.6417 \mathrm{e}-14$ & $2.1473 \mathrm{e}-13$ \\
\hline
\end{tabular}


Table 4.2 Maximum absolute residual errors obtained by direct Haar wavelet for fixed values of $\delta=0.5 \epsilon, \eta=0.5 \epsilon$ and different values of $\epsilon$.

\begin{tabular}{cccccl}
\hline$\epsilon \backslash N$ & 32 & 64 & 128 & 256 & 512 \\
\hline $5^{-1}$ & $3.6082 \mathrm{e}-16$ & $1.1102 \mathrm{e}-16$ & $1.4572 \mathrm{e}-16$ & $1.0408 \mathrm{e}-16$ & $1.3704 \mathrm{e}-16$ \\
$5^{-2}$ & $1.3600 \mathrm{e}-15$ & $9.8532 \mathrm{e}-16$ & $1.0408 \mathrm{e}-15$ & $1.0183 \mathrm{e}-15$ & $6.5052 \mathrm{e}-16$ \\
$5^{-3}$ & $3.2474 \mathrm{e}-15$ & $5.4540 \mathrm{e}-15$ & $7.6605 \mathrm{e}-15$ & $6.8157 \mathrm{e}-15$ & $3.9682 \mathrm{e}-15$ \\
$5^{-4}$ & $2.3453 \mathrm{e}-15$ & $2.6160 \mathrm{e}-15$ & $1.4613 \mathrm{e}-14$ & $8.5956 \mathrm{e}-15$ & $2.1762 \mathrm{e}-14$ \\
\hline
\end{tabular}

Table 4.3 Maximum absolute residual errors obtained in [29] for fixed values of $\delta=0.5 \epsilon, \eta=0.5 \epsilon$ and different values of $\epsilon$.

\begin{tabular}{ccccccc}
\hline$\epsilon \backslash N$ & 64 & 128 & 256 & 512 & 1024 & 2048 \\
\hline $5^{-1}$ & $2.200 \mathrm{e}-03$ & $5.600 \mathrm{e}-04$ & $1.400 \mathrm{e}-04$ & $3.500 \mathrm{e}-05$ & $8.700 \mathrm{e}-06$ & $2.200 \mathrm{e}-06$ \\
$5^{-2}$ & $1.900 \mathrm{e}-02$ & $4.800 \mathrm{e}-03$ & $1.200 \mathrm{e}-03$ & $3.000 \mathrm{e}-04$ & $7.400 \mathrm{e}-05$ & $1.900 \mathrm{e}-05$ \\
$5^{-3}$ & $9.700 \mathrm{e}-02$ & $2.400 \mathrm{e}-02$ & $6.100 \mathrm{e}-03$ & $1.500 \mathrm{e}-03$ & $3.800 \mathrm{e}-04$ & $9.600 \mathrm{e}-05$ \\
$5^{-4}$ & $4.400 \mathrm{e}-01$ & $1.200 \mathrm{e}-01$ & $3.000 \mathrm{e}-02$ & $7.600 \mathrm{e}-03$ & $1.900 \mathrm{e}-03$ & $4.700 \mathrm{e}-04$ \\
$10^{-5}$ & $7.600 \mathrm{e}-03$ & $2.000 \mathrm{e}-03$ & $5.400 \mathrm{e}-04$ & $1.400 \mathrm{e}-04$ & $3.500 \mathrm{e}-05$ & $8.80 \mathrm{e}-06$ \\
$10^{-6}$ & $7.600 \mathrm{e}-03$ & $2.000 \mathrm{e}-03$ & $5.400 \mathrm{e}-04$ & $1.400 \mathrm{e}-04$ & $3.500 \mathrm{e}-05$ & $8.80 \mathrm{e}-06$ \\
$10^{-7}$ & $7.600 \mathrm{e}-03$ & $2.000 \mathrm{e}-03$ & $5.400 \mathrm{e}-04$ & $1.400 \mathrm{e}-04$ & $3.500 \mathrm{e}-05$ & $8.800 \mathrm{e}-06$ \\
$10^{-8}$ & $7.600 \mathrm{e}-03$ & $2.000 \mathrm{e}-03$ & $5.400 \mathrm{e}-04$ & $1.400 \mathrm{e}-04$ & $3.500 \mathrm{e}-05$ & $8.800 \mathrm{e}-06$ \\
$10^{-9}$ & $7.600 \mathrm{e}-03$ & $2.000 \mathrm{e}-03$ & $5.400 \mathrm{e}-04$ & $1.400 \mathrm{e}-04$ & $3.500 \mathrm{e}-05$ & $8.800 \mathrm{e}-06$ \\
$10^{-10}$ & $7.600 \mathrm{e}-03$ & $2.000 \mathrm{e}-03$ & $5.400 \mathrm{e}-04$ & $1.400 \mathrm{e}-04$ & $3.500 \mathrm{e}-05$ & $8.800 \mathrm{e}-06$ \\
$10^{-11}$ & $7.600 \mathrm{e}-03$ & $2.000 \mathrm{e}-03$ & $5.400 \mathrm{e}-04$ & $1.400 \mathrm{e}-04$ & $3.500 \mathrm{e}-05$ & $8.800 \mathrm{e}-06$ \\
$10^{-12}$ & $7.600 \mathrm{e}-03$ & $2.000 \mathrm{e}-03$ & $5.400 \mathrm{e}-04$ & $1.400 \mathrm{e}-04$ & $3.500 \mathrm{e}-05$ & $8.800 \mathrm{e}-06$ \\
$10^{-13}$ & $7.600 \mathrm{e}-03$ & $2.000 \mathrm{e}-03$ & $5.400 \mathrm{e}-04$ & $1.400 \mathrm{e}-04$ & $3.500 \mathrm{e}-05$ & $8.800 \mathrm{e}-06$ \\
$10^{-14}$ & $7.600 \mathrm{e}-03$ & $2.000 \mathrm{e}-03$ & $5.400 \mathrm{e}-04$ & $1.400 \mathrm{e}-04$ & $3.500 \mathrm{e}-05$ & $8.800 \mathrm{e}-06$ \\
$10^{-15}$ & $7.600 \mathrm{e}-03$ & $2.000 \mathrm{e}-03$ & $5.400 \mathrm{e}-04$ & $1.400 \mathrm{e}-04$ & $3.500 \mathrm{e}-05$ & $8.800 \mathrm{e}-06$ \\
$10^{-16}$ & $7.600 \mathrm{e}-03$ & $2.000 \mathrm{e}-03$ & $5.400 \mathrm{e}-04$ & $1.400 \mathrm{e}-04$ & $3.500 \mathrm{e}-05$ & $8.800 \mathrm{e}-06$ \\
\hline
\end{tabular}

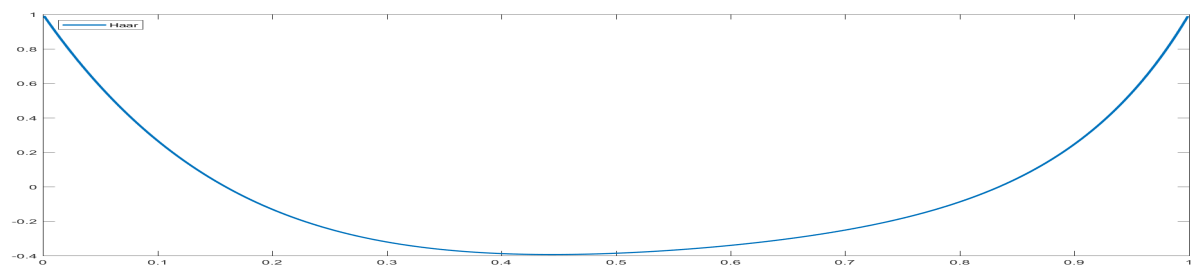

Figure 10. Haar wavelet solution of problem 4 for $\epsilon=5^{-2}, \delta=0.5 \epsilon$ and $\eta=0.5 \epsilon$ with $J=8$. 


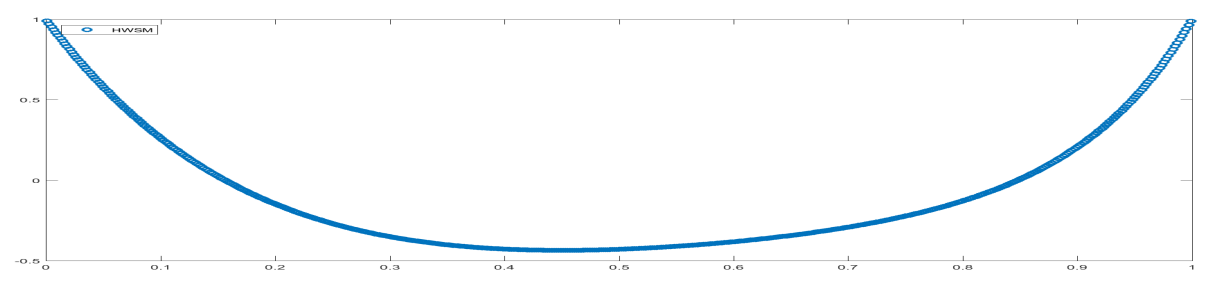

Figure 11. Direct Haar wavelet solution of problem 4 for $\epsilon=5^{-2}, \delta=0.5 \epsilon$ and $\eta=0.5 \epsilon$ with $J=8$.

Problem 5. Let us consider the following SPDDE with term containing both delay and shift

$$
\epsilon^{2} y^{\prime \prime}(t)-e^{0.5} y(t-\delta)+t y(t)-\left(1+t^{2}\right) y(t+\eta)=1, \quad t \in(0,1)
$$

with boundary conditions

$$
y(0)=1, \text { on } \quad-\delta \leq t \leq 0, \quad y(1)=1, \quad 1 \leq t \leq 1+\eta
$$

The exact solution is not known.

Maximum absolute residual errors (MARE) for a particular value of perturbation parameter and various values of delay and shift parameters obtained by Haar wavelet method with different resolutions level is given in the table 5.1. MARE obtained by direct Haar wavelet method is given in the table 5.2 and non standard finite difference method [29] is given in the table 5.3. Also, graph of Haar wavelet solution is given in figures 12 and 13.

Table 5.1 Maximum absolute residual errors obtained by Haar wavelet for fixed values of $\delta=0.5 \epsilon, \eta=0.5 \epsilon$ and different values of $\epsilon$.

\begin{tabular}{cccccccc}
\hline$\epsilon \backslash N$ & 32 & 64 & 128 & 256 & 512 & 1024 & 2048 \\
\hline $5^{-1}$ & $7.0777 \mathrm{e}-16$ & $4.7878 \mathrm{e}-16$ & $3.5735 \mathrm{e}-16$ & $1.7868 \mathrm{e}-16$ & $1.9516 \mathrm{e}-16$ & $1.4181 \mathrm{e}-16$ & $1.3119 \mathrm{e}-16$ \\
$5^{-2}$ & $1.4710 \mathrm{e}-15$ & $9.9226 \mathrm{e}-16$ & $1.5474 \mathrm{e}-15$ & $1.1848 \mathrm{e}-15$ & $1.2655 \mathrm{e}-15$ & $1.1332 \mathrm{e}-15$ & $1.1130 \mathrm{e}-15$ \\
$5^{-3}$ & $8.4655 \mathrm{e}-16$ & $2.4217 \mathrm{e}-15$ & $4.7636 \mathrm{e}-15$ & $6.4219 \mathrm{e}-15$ & $9.0457 \mathrm{e}-15$ & $2.0899 \mathrm{e}-14$ & $4.5240 \mathrm{e}-14$ \\
$5^{-4}$ & $2.3176 \mathrm{e}-15$ & $5.6691 \mathrm{e}-15$ & $4.4305 \mathrm{e}-15$ & $1.4240 \mathrm{e}-14$ & $1.8427 \mathrm{e}-14$ & $1.8345 \mathrm{e}-14$ & $8.8708 \mathrm{e}-14$ \\
$10^{-5}$ & $2.0400 \mathrm{e}-15$ & $2.1996 \mathrm{e}-15$ & $4.8746 \mathrm{e}-15$ & $1.3907 \mathrm{e}-14$ & $2.5588 \mathrm{e}-14$ & $9.5617 \mathrm{e}-14$ & $2.5931 \mathrm{e}-13$ \\
$10^{-6}$ & $1.9568 \mathrm{e}-15$ & $2.8658 \mathrm{e}-15$ & $4.8746 \mathrm{e}-15$ & $8.7586 \mathrm{e}-15$ & $2.4811 \mathrm{e}-14$ & $8.6735 \mathrm{e}-14$ & $2.6142 \mathrm{e}-13$ \\
$10^{-7}$ & $1.9013 \mathrm{e}-15$ & $5.9744 \mathrm{e}-15$ & $5.3395 \mathrm{e}-15$ & $9.2201 \mathrm{e}-15$ & $2.4256 \mathrm{e}-14$ & $8.6957 \mathrm{e}-14$ & $2.7199 \mathrm{e}-13$ \\
$10^{-8}$ & $1.7347 \mathrm{e}-15$ & $3.0184 \mathrm{e}-15$ & $4.4097 \mathrm{e}-15$ & $9.6641 \mathrm{e}-15$ & $2.5366 \mathrm{e}-14$ & $8.7903 \mathrm{e}-14$ & $2.5986 \mathrm{e}-13$ \\
$10^{-9}$ & $1.3739 \mathrm{e}-15$ & $1.7972 \mathrm{e}-15$ & $3.3411 \mathrm{e}-15$ & $8.5539 \mathrm{e}-15$ & $2.4700 \mathrm{e}-14$ & $8.7179 \mathrm{e}-14$ & $2.9661 \mathrm{e}-13$ \\
$10^{-10}$ & $2.4009 \mathrm{e}-15$ & $3.5735 \mathrm{e}-15$ & $3.1329 \mathrm{e}-15$ & $8.9078 \mathrm{e}-15$ & $2.4700 \mathrm{e}-14$ & $9.7615 \mathrm{e}-14$ & $2.5964 \mathrm{e}-13$ \\
$10^{-11}$ & $1.3739 \mathrm{e}-15$ & $5.2111 \mathrm{e}-15$ & $3.7852 \mathrm{e}-15$ & $1.6464 \mathrm{e}-14$ & $2.4589 \mathrm{e}-14$ & $8.7401 \mathrm{e}-14$ & $3.0316 \mathrm{e}-13$ \\
$10^{-12}$ & $1.9568 \mathrm{e}-15$ & $3.1294 \mathrm{e}-15$ & $3.5631 \mathrm{e}-15$ & $1.0108 \mathrm{e}-14$ & $2.7475 \mathrm{e}-14$ & $8.6291 \mathrm{e}-14$ & $2.5908 \mathrm{e}-13$ \\
$10^{-13}$ & $2.0400 \mathrm{e}-15$ & $2.4633 \mathrm{e}-15$ & $4.0072 \mathrm{e}-15$ & $1.2218 \mathrm{e}-14$ & $2.6143 \mathrm{e}-14$ & $8.6291 \mathrm{e}-14$ & $2.6519 \mathrm{e}-13$ \\
$10^{-14}$ & $1.1519 \mathrm{e}-15$ & $5.9328 \mathrm{e}-15$ & $3.1190 \mathrm{e}-15$ & $1.3869 \mathrm{e}-14$ & $2.5699 \mathrm{e}-14$ & $8.7845 \mathrm{e}-14$ & $2.9439 \mathrm{e}-13$ \\
$10^{-15}$ & $1.7347 \mathrm{e}-15$ & $3.4764 \mathrm{e}-15$ & $4.2293 \mathrm{e}-15$ & $8.5435 \mathrm{e}-15$ & $2.4145 \mathrm{e}-14$ & $8.9177 \mathrm{e}-14$ & $3.0971 \mathrm{e}-13$ \\
$10^{-16}$ & $1.5127 \mathrm{e}-15$ & $2.1996 \mathrm{e}-15$ & $3.5631 \mathrm{e}-15$ & $1.5496 \mathrm{e}-14$ & $2.5699 \mathrm{e}-14$ & $8.7179 \mathrm{e}-14$ & $2.6197 \mathrm{e}-13$ \\
\hline
\end{tabular}


Table 5.2 Maximum absolute residual errors obtained by direct Haar wavelet for fixed values of $\delta=0.5 \epsilon, \eta=0.5 \epsilon$ and different values of $\epsilon$.

\begin{tabular}{cccccl}
\hline$\epsilon \backslash N$ & 32 & 64 & 128 & 256 & 512 \\
\hline $5^{-1}$ & $2.2204 \mathrm{e}-16$ & $2.0817 \mathrm{e}-16$ & $1.2837 \mathrm{e}-16$ & $1.2143 \mathrm{e}-16$ & $7.8063 \mathrm{e}-17$ \\
$5^{-2}$ & $1.2629 \mathrm{e}-15$ & $1.6515 \mathrm{e}-15$ & $2.1927 \mathrm{e}-15$ & $1.0165 \mathrm{e}-15$ & $1.0009 \mathrm{e}-15$ \\
$5^{-3}$ & $2.6368 \mathrm{e}-15$ & $3.2058 \mathrm{e}-15$ & $1.7916 \mathrm{e}-14$ & $5.3534 \mathrm{e}-15$ & $7.8878 \mathrm{e}-15$ \\
$5^{-4}$ & $3.3445 \mathrm{e}-15$ & $2.8658 \mathrm{e}-15$ & $7.1505 \mathrm{e}-15$ & $1.0349 \mathrm{e}-14$ & $2.5159 \mathrm{e}-14$ \\
\hline
\end{tabular}

Table 5.3 Maximum absolute residual errors obtained in [29] for fixed values of $\delta=0.5 \epsilon, \eta=0.5 \epsilon$ and different values of $\epsilon$.

\begin{tabular}{ccccccc}
\hline$\epsilon \backslash N$ & 64 & 128 & 256 & 512 & 1024 & 2048 \\
\hline $5^{-1}$ & $2.200 \mathrm{e}-02$ & $5.500 \mathrm{e}-03$ & $1.400 \mathrm{e}-03$ & $3.500 \mathrm{e}-04$ & $8.600 \mathrm{e}-05$ & $2.200 \mathrm{e}-05$ \\
$5^{-2}$ & $4.800 \mathrm{e}-01$ & $1.300 \mathrm{e}-01$ & $3.200 \mathrm{e}-02$ & $8.100 \mathrm{e}-02$ & $2.000 \mathrm{e}-03$ & $5.100 \mathrm{e}-04$ \\
$5^{-3}$ & $1.200 \mathrm{e}-02$ & $1.400 \mathrm{e}-01$ & $6.800 \mathrm{e}-01$ & $1.900 \mathrm{e}-01$ & $4.800 \mathrm{e}-02$ & $1.200 \mathrm{e}-02$ \\
$5^{-4}$ & $2.000 \mathrm{e}-02$ & $2.000 \mathrm{e}-01$ & $8.400 \mathrm{e}-01$ & $1.500 \mathrm{e}-01$ & $9.400 \mathrm{e}-01$ & $2.700 \mathrm{e}-01$ \\
$10^{-5}$ & $9.300 \mathrm{e}-03$ & $2.100 \mathrm{e}-02$ & $4.300 \mathrm{e}-02$ & $8.700 \mathrm{e}-02$ & $1.700 \mathrm{e}-01$ & $3.500 \mathrm{e}-01$ \\
$10^{-6}$ & $3.200 \mathrm{e}-03$ & $1.800 \mathrm{e}-03$ & $4.200 \mathrm{e}-03$ & $8.600 \mathrm{e}-03$ & $1.700 \mathrm{e}-02$ & $3.500 \mathrm{e}-02$ \\
$10^{-7}$ & $3.200 \mathrm{e}-03$ & $8.000 \mathrm{e}-04$ & $3.400 \mathrm{e}-03$ & $8.400 \mathrm{e}-04$ & $1.700 \mathrm{e}-03$ & $3.500 \mathrm{e}-03$ \\
$10^{-8}$ & $3.200 \mathrm{e}-03$ & $8.000 \mathrm{e}-04$ & $2.000 \mathrm{e}-04$ & $6.200 \mathrm{e}-05$ & $1.700 \mathrm{e}-04$ & $3.400 \mathrm{e}-04$ \\
$10^{-9}$ & $3.200 \mathrm{e}-03$ & $8.000 \mathrm{e}-04$ & $2.000 \mathrm{e}-04$ & $5.000 \mathrm{e}-05$ & $1.200 \mathrm{e}-05$ & $3.400 \mathrm{e}-05$ \\
$10^{-10}$ & $3.200 \mathrm{e}-03$ & $8.000 \mathrm{e}-04$ & $2.000 \mathrm{e}-04$ & $5.000 \mathrm{e}-05$ & $1.200 \mathrm{e}-05$ & $3.100 \mathrm{e}-06$ \\
$10^{-11}$ & $3.200 \mathrm{e}-03$ & $8.000 \mathrm{e}-04$ & $2.000 \mathrm{e}-04$ & $5.000 \mathrm{e}-05$ & $1.200 \mathrm{e}-05$ & $3.100 \mathrm{e}-06$ \\
$10^{-12}$ & $3.200 \mathrm{e}-03$ & $8.000 \mathrm{e}-04$ & $2.000 \mathrm{e}-04$ & $5.000 \mathrm{e}-05$ & $1.200 \mathrm{e}-05$ & $3.100 \mathrm{e}-06$ \\
$10^{-13}$ & $3.200 \mathrm{e}-03$ & $8.000 \mathrm{e}-04$ & $2.000 \mathrm{e}-04$ & $5.000 \mathrm{e}-05$ & $1.200 \mathrm{e}-05$ & $3.100 \mathrm{e}-06$ \\
$10^{-14}$ & $3.200 \mathrm{e}-03$ & $8.000 \mathrm{e}-04$ & $2.000 \mathrm{e}-04$ & $5.000 \mathrm{e}-05$ & $1.200 \mathrm{e}-05$ & $3.100 \mathrm{e}-06$ \\
$10^{-15}$ & $3.200 \mathrm{e}-03$ & $8.000 \mathrm{e}-04$ & $2.000 \mathrm{e}-04$ & $5.000 \mathrm{e}-05$ & $1.200 \mathrm{e}-05$ & $3.100 \mathrm{e}-06$ \\
$10^{-16}$ & $3.200 \mathrm{e}-03$ & $8.000 \mathrm{e}-04$ & $2.000 \mathrm{e}-04$ & $5.000 \mathrm{e}-05$ & $1.200 \mathrm{e}-05$ & $3.100 \mathrm{e}-06$ \\
\hline
\end{tabular}

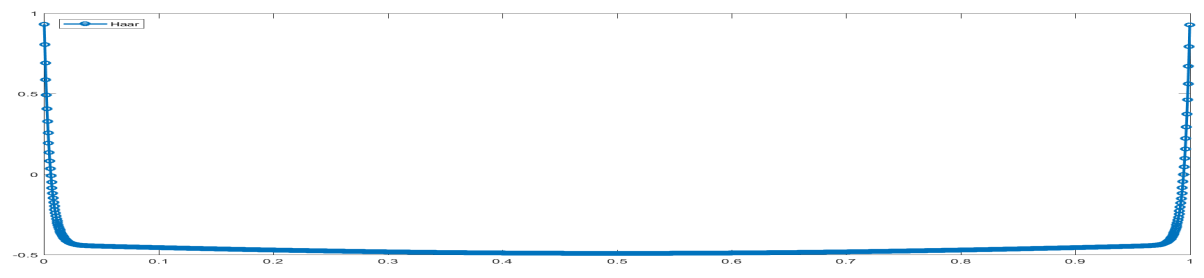

Figure 12. Haar wavelet solution of problem 5 for $\epsilon=\frac{1}{5^{2}}$ and $\delta=0.5 \epsilon, \eta=0.5 \epsilon$ with $J=10$. 


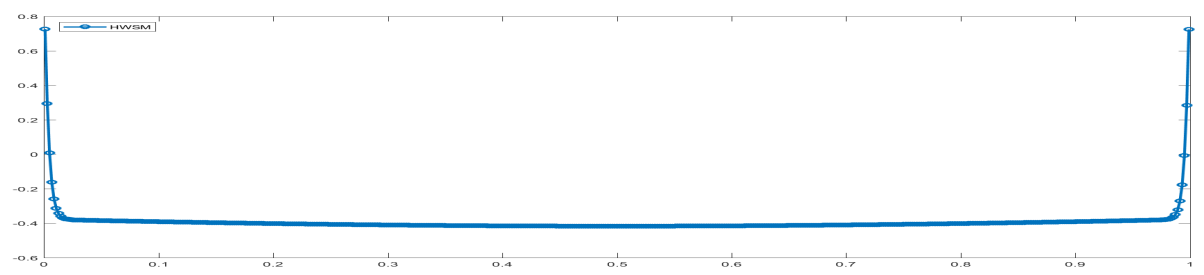

Figure 13. Direct Haar wavelet solution of problem 5 for $\epsilon=\frac{1}{5^{3}}$ and $\delta=0.5 \epsilon, \eta=0.5 \epsilon$ with $J=8$.

\section{Conclusion}

We have converted delay and shift terms using Taylor series expansion and then we applied Haar wavelet method which transforms the linear singularly perturbed differential equations with delay and shift into system of linear equations and then, we obtain the approximate solution. Further we solved linear singularly perturbed differential equations with delay and shift using Haar wavelet directly. Both the methods yield almost same results when the delayed and shift terms are very small. In case if delayed and shift terms are large ( not depending on $\epsilon$ ) then direct Haar wavelet series method is efficient to solve the delay and shift term. For large delay or neutral delay we refer to [30] by Raza and Khan. The results presented in the tables show that error stabilized after some certain $\epsilon$, which shows that the method is independent of $\epsilon$. We have also compared our results with the results of existing methods given in [11, 29], which are tabulated in the tables 1.1-1.4, 2.1-2.4, 3.1-3.7, 4.1-4.3 and 5.1-5.3 which clearly indicate that Haar wavelet produces better results in comparison of finite difference and fitted operator finite difference method. Further the graphs of solved examples have been demonstrated in the Figures 1-13. The technique introduced here is easy to apply as well as the performance of the present method yields more accurate results on increasing the resolution level.

\section{Acknowledgments}

The authors would like to express their sincere thanks to reviewer for his valuable suggestions which greatly improved the quality of the paper.

Second author is very thankful to the UGC, New Delhi, for providing the financial support as SRF.

\section{References}

[1] K. Ahmad and F. A. Shah, Introduction to Wavelets with Applications, Real World Education Publishers, New Delhi (2013).

[2] T. Aziz and A. Khan, A spline method for second-order singularly perturbed boundary-value problems, J. Comput. Appl. Math., 147, 445-452 (2002).

[3] C. F. Chen and C. H. Hsiao, Haar wavelet method for solving lumped and distributedparameter system, IEEE Proc.-Control Theory Appl., 144, 87-94 (1997). 
[4] I. Daubechies, Orthonormal bases of compactly supported wavelets, Comm. Pure Appl. Math. 41, 909-996 (1988).

[5] L. Debnath and F. A. Shah, Wavelet transform and their application, Springer New York, Birkhauser, 337-440 (2015).

[6] M. Frazier, B. Jawerth and G. Weiss, Littlewood-Paley Theory and the Study of Function Spaces, CBMS Regional Conference Series in Mathematics (The American Mathematical Society, Providence, RI), 79, (1991).

[7] A. Haar, Zur theorie der orthogonalen funktionen-systeme, Math. Ann. 69, 331-371 (1910).

[8] E. Hernandez and G. Weiss, A First Course on Wavelets, Studies in Advanced Mathematics, CRC Press, Boca Raton, FL, (1996).

[9] S. Islam, I. Aziz and B. Sarler, The numerical solution of second order boundary value problems by collocation method with Haar wavelets, Math Comput. Model, 50, 1577-1590 (2010).

[10] M. K. Kadalbajoo and K. K. Sharma, Numerical analysis of boundary-value problems for singularly perturbed differential-difference equations: small shifts of mixed type with rapid oscillations, Commun. Numer. Meth. Engng 20, 167182 (2004).

[11] M. K. Kadalbajoo and K. K. Sharma, Numerical analysis of boundary-value problems for singularly-perturbed differential-difference equations with small shifts of mixed type, J. Opt. Theory Appl. 115, 145-163 (2002).

[12] M. K. Kadalbajoo and K. K. Sharma, Numerical treatment of a mathematical model arising from a model of neuronal variability, J. Math. Anal. Appl. 307, 606-627 (2005).

[13] A. Khan and P. Khandelwal, Non-polynomial sextic spline solution of singularly perturbed boundary-value problems, Int. J. Comp. Math., 91, 1122-1135 (2014).

[14] A. Khan, I. Khan and T. Aziz, Sextic spline solution of singularly perturbed boundary-value problems, Appl. Math. Comput. 181, 432-439 (2006).

[15] J. Kevorkian and J. D. Cole, Perturbation Methods in Applied Mathematics, Springer, New York. Mathematics, 20, 33-53 (1981).

[16] D. Kumar and M. K. Kadalbajoo, A parameter-uniform numerical method for timedependent singularly perturbed differential-difference equations, Appl. Math. Model. 35, 2805-2819 (2011).

[17] D. Kumar and M. K. Kadalbajoo, Numerical treatment of singularly perturbed delay differential equations using B-Spline collocation method on Shishkin mesh, J. Numer. Anal. Indust. App. Math., 7, 73-90 (2012).

[18] D. Kumar and M. K. Kadalbajoo, Numerical approximations for singularly perturbed differential-difference BVPs with layer and oscillatory behaviour, J. Numer. Math., 20, 33-53 (2012).

[19] V. Kumar and M. Mehra, Cubic spline adaptive wavelet scheme to solve singularly perturbed reaction-diffusion problems, J. Wavelets Multiresoult. Inf. Process. 5(2), 317-331 (2007). 
[20] C. G. Lange and R. M. Miura, Singular perturbation analysis of boundary-value problems for differential-difference equations. v. small shifts with layer behavior, SIAM J. Appl. Math. 54, 249-272 ( 1994).

[21] U. Lepik, Application of Haar wavelet transform to solving integral and differential equations, Appl. Math. Comput. 57, 28-46 (2007).

[22] U. Lepik, Haar wavelet method for solving stiff differential equations, Math. Mode. Anal. 14, 467-481 (2009).

[23] U. Lepik and H. Hein, Haar Wavelet With Applications, Springer, 7-44 (2014).

[24] S. G. Mallat, Multiresolution approximations and wavelet orthonormal bases of $L^{2}(R)$, Trans. Amer. Math. Soc. 315, 69-87 (1989).

[25] A. H. Nayfeh, Perturbation Methods, Wiley, New York (1979).

[26] O. Oruc, A Haar wavelet approximation for two-dimensional time fractional reactionsubdiffusion equation, Engineering Computers. https://doi.org/10.1007/s00366-018-0584-8 (2018).

[27] S. Pandit and M. Kumar, Haar wavelet approach for numerical solution of two parameters singularly perturbed boundary value problems. Appl. Math. Inf. Sci., 8, 2965-2974 (2014).

[28] S. Pandit and M. Kumar, Wavelet Transform and Wavelet-based Numerical Methods: Int. J. Nonlinear Sci. 13, 325-345 (2012).

[29] K. C. Patidar and K. K. Sharma, Uniformly convergent non-standard finite difference methods for singularly perturbed differential-difference equation with delay and advance, Int. J. Numer. Meth. Engng. 66, 272-296 (2006).

[30] A. Raza and A. Khan, Haar wavelet series solution for solving neutral delay differential equations, J. King Saud Uni.-Sci., 31, 1070-1076 (2019). https://doi.org/10.1016/j.jksus.2018.09.013 (2019).

[31] P. Rai and K. K. Sharma, Numerical study of singularly perturbed differential-difference equation arising in the modeling of neuronal variability, Comput. Math. Appl. 63, 118-132 (2012).

[32] F. A. Shah, R. Abass and J. Iqbal, Numerical solution of singularly perturbed problems using Haar wavelet collocation method, Cogent Math. 3, (2016).

[33] F. A. Shah and R. Abass, An efficient wavelet-based collocation method for handling singularly perturbed boundary-value problems in fluid mechanics, Int. J. Non. Sci. and Num. Sim., 18(6), 7-25 (2017).

Arshad Khan Department of Mathematics, Jamia Millia Islamia, New Delhi-110025, India E-mail: akhan2@jmi.ac.in

Akmal Raza Department of Mathematics, Jamia Millia Islamia, New Delhi-110025, India E-mail: akmalrazataqvi@gmail.com 